\title{
Terahertz Components Packaging Using Integrated Waveguide Technology
}

\author{
V. Desmaris, D. Meledin, D. Dochev, A. Pavolotsky and V. Belitsky \\ Group for Advanced Receiver Development, Earth and Space Science Department, \\ Chalmers University of Technology, Gothenburg, Sweden
}

\begin{abstract}
We present an integrated waveguide based packaging solution compatible with different $\mathrm{THz}$ component technologies, both for room temperature and cryogenic operations, employing space-qualified wire-bonding for electrical contacts. The proposed waveguide packaging relies on the combination of all-metal micro-machined $\mathrm{THz}$ waveguide and active component chip layouts suitable for the realization of systems from 200 up to $5000 \mathrm{GHz}$. It provides possibility of making 3-dimensional structures via facilitating of multi-level (layered) designs. The surface roughness of the fabricated $\mathrm{THz}$ waveguide structure was demonstrated to be $20 \mathrm{~nm}$, while a $2 \mu \mathrm{m}$ alignment accuracy of the active component chip was verified.

Index Terms - Submillimeter wave technology, Packaging, Submillimeter wave devices, Integrated circuits packaging.
\end{abstract}

\section{INTRODUCTION}

In order to produce complete systems, the $\mathrm{THz}$ components, independently of their core technology (e.g, Schottky diodes, MMIC, SIS or HEB mixers), have to be integrated into $\mathrm{THz}$ electromagnetic guiding structures, which in this context are defined as $\mathrm{THz}$ packaging.

Waveguides are already widely used in the industry but mainly at microwave $(<100 \mathrm{GHz})$ frequencies. However, waveguides are particularly attractive guiding systems for electromagnetic waves, since they are relatively broadband and low loss transmission lines. Furthermore, waveguides provides complete confinement of the EM field inside a hollow structure and thus have negligible sensitivity to RF interference and limit RFI/EMI in the systems.

The challenges for waveguide at $\mathrm{THz}$ frequencies are the small waveguide dimensions $(\sim 100 \times 200 \mu \mathrm{m} 2$ and $\sim 20 \times 40$ $\mu \mathrm{m} 2$ at $1 \mathrm{THz}$ and $5 \mathrm{THz}$, respectively) and the required nanometric surface roughness of the waveguide walls. The small dimensions originate from the scaling of the solutions of Maxwell's equation with the wavelength. The surface roughness limitations are in turn coupled to the submicron size of the skin depth at these frequencies, and directly responsible for the RF losses. In any case, both these constraints are completely out of reach for conventional machining even with the latest $\mathrm{CNC}$ technology, even though slightly more tolerant designs of components have been introduced for hybrids [1].

A considerable difference between microwave components and $\mathrm{THz}$ components lies in their size. As a consequence, handling the substrate-based active components and mounting them into a waveguide structure becomes really demanding at $\mathrm{THz}$ frequencies when the dimensions of such "substrate" becomes as small as e.g, 1000x $70 \times 17 \mu \mathrm{m}[2]$.

In this paper, we present a complete waveguide packaging technology suitable for frequencies up to $5000 \mathrm{GHz}$, combining all-metal $\mathrm{THz}$ waveguide technology [3] based on micromachining, for ultimate waveguide quality and a novel chip layout forming a $\Pi$-frame made of SOI structure [4], supporting the active devices on a thin cross beam, for simple, fast and accurate chip positioning.

\section{All Metal Waveguide Technology}

Multi-level (layered) waveguide structures were fabricated using the GARD process [3],[5], for different SIS and HEB mixers for frequencies ranging from $350 \mathrm{GHz}$ to $2 \mathrm{THz}$ [4],[6]-[7] (Fig.1).

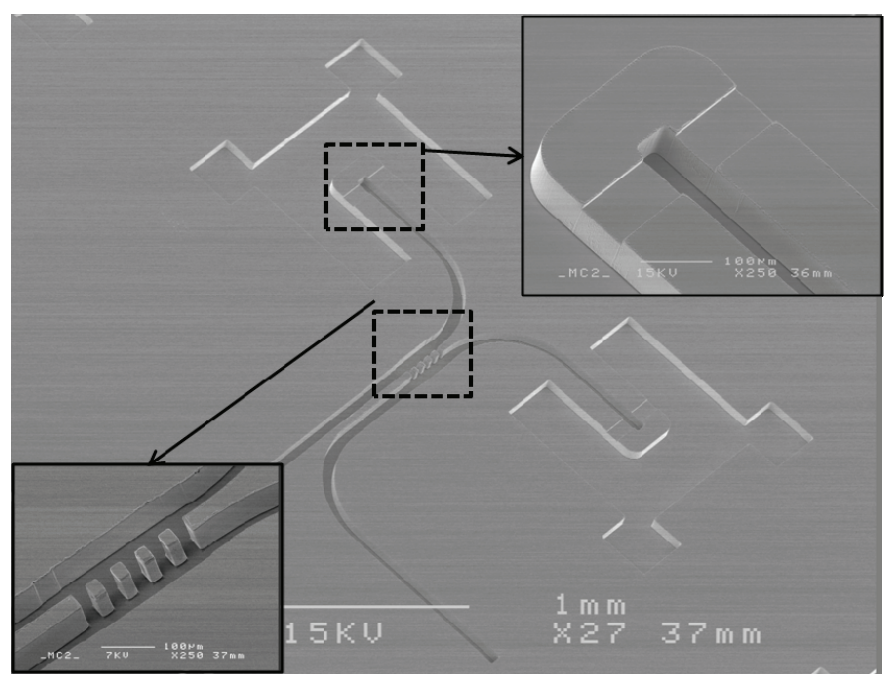

Fig. 1. Waveguide circuits of a balanced HEB mixer operating in the 1.6-2 THz frequency range.

The process is based on copper electroforming over a dissolvable layered mold made out of SU- 8 and pattern by conventional photolithography. As a result, submicron linear accuracy and surface roughness of the waveguide walls down to $20 \mathrm{~nm}$ were verified [4] on the fabricated waveguide structures. Furthermore, the fabricated hardware is already in use on the SHeFI receiver of the APEX telescope [2],[6]. 
Importantly, the photolithographic nature of the GARD process makes this fabrication technique very attractive for volume production due to its throughput and reproducibility, compared to the competing technologies.

The availability of split block technology at $\mathrm{THz}$ frequencies, along with achieving extremely high waveguide surface quality and thus low RF loss and possibility of using multi-level designs, opens solid prospects for building complex waveguide circuits. This naturally increases leads to an increase of integration level in $\mathrm{THz}$ systems, making them more compact and eventually lightweight, which might be very relevant for space borne applications.

The low loss properties of the all-metal waveguide have been largely demonstrated at the system level on cryogenic receivers. We intend to present additional characterization of bare waveguide structures, through direct S-parameter measurements at room temperature, in order to demonstrate further that this technology is not limited to low temperature hardware. In fact, it is fully compatible with room temperature $\mathrm{THz}$ and sub-millimeter wave technologies, MMICs or Schottky diodes.

\section{THz COMPONENT INTEGRATION}

For the demonstration of our packaging solution, we carried out 3D FEM electromagnetic simulations of a split block waveguide balanced HEB mixer for the frequency range 1.6-2 THz. The simulations resulted in a mixer chip dimensions of $360 \times 50 \times 2 \mu \mathrm{m}$. Such chip would clearly extremely difficult to handle and mount in the mixer block [4].

In our novel approach, starting from SOI wafers, a Пshaped bulk silicon frame is formed, providing alignment reference for the active device fabricated on a cross beam (Fig. 2). The shape of the HEB mixer chip is defined via micromachining, photolithography and consequent etching, while the thickness of the beam and the supporting frame depends on the SOI substrate. Consequently, the integration of the HEB mixer chips having this novel layout into the mixer block does not require any additional lapping and dicing.

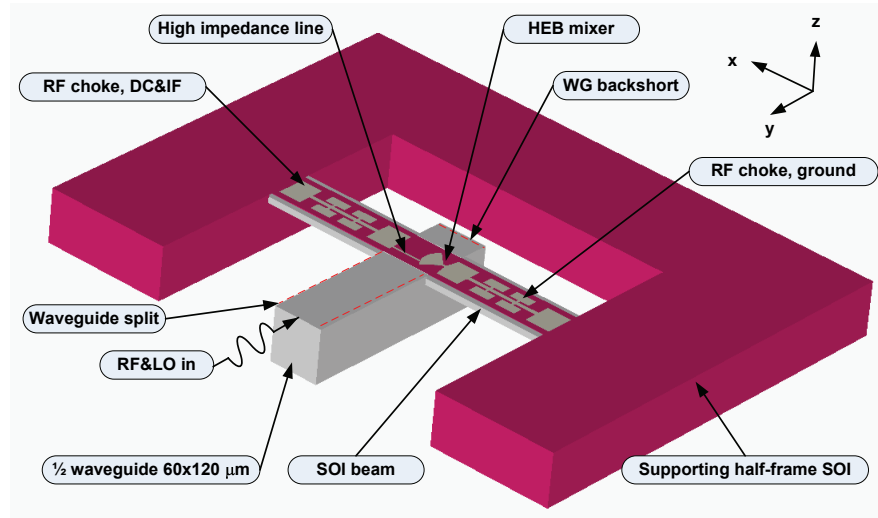

Fig. 2. Illustration of the HEB mixer chip with supporting halfframe [4].
With this chip layout, the shape and the dimensions of the supporting $\Pi$-frame are chosen such that it provides alignment reference with respect to the corresponding recess in the mixer housing where it should be integrated. Based on this approach we have fabricated a technology demonstrator for $1.6-2.0$ $\mathrm{THz}$ receiver (Fig 3.), where an alignment accuracy of in the order of 2 micrometers has been confirmed.

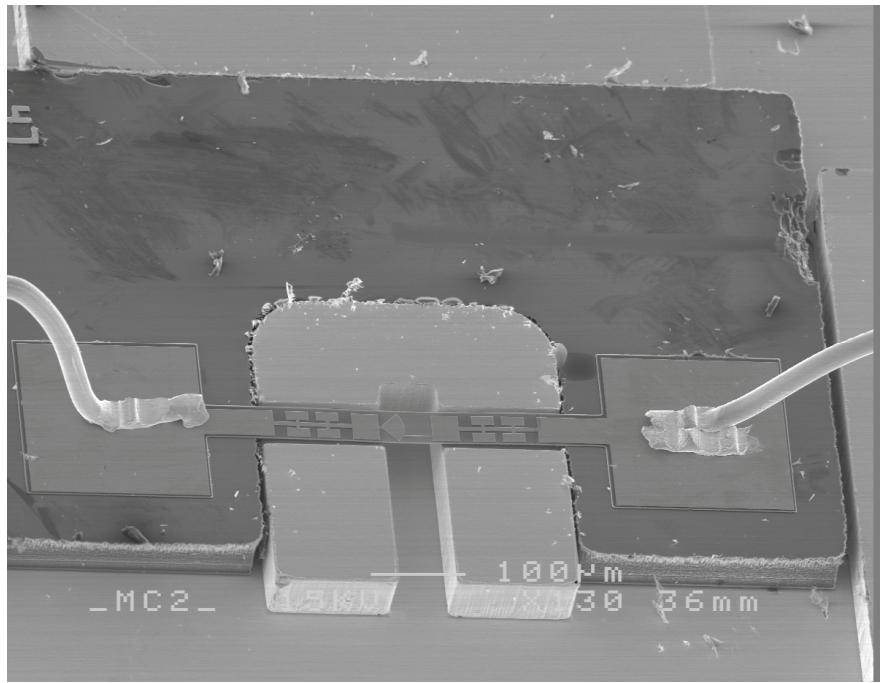

Fig. 3. HEB chip in all waveguide metal housing, bonded for sole purpose of demonstration [4].

\section{CONCLUSIONS}

In this paper, present a complete waveguide packaging technology, suitable for all millimeter and $\mathrm{THz}$ device technology with the considerable advantage of being reliably compatible with cryogenic operating conditions. The presented packaging technology takes full advantages of allmetal waveguide combined with a novel component design. This design uses a novel layout for the active devices employing bulk $\mathrm{Si} \Pi$-frame, supporting a cross beam accommodating the active devices. We successfully demonstrated all technological steps and final integration of a balanced $\mathrm{THz}$ mixer. We believe that the demonstrated approach is suitable for building a single-end deep-terahertz mixer operating at up to $5 \mathrm{THz}$. The confirmed ease of integration by means of self-aligning of the mixer chip in the mixer housing opens prospective for making moderately large heterodyne terahertz array systems

\section{ACKNOWLEDGEMENT}

Erik Sundin (GARD) is acknowledged for his help during the DC characterization. This work was supported by the European Commission Framework Programme 7, Advanced Radio Astronomy in Europe RadioNet, via JRA AMSTAR+, Grant No. 227290. 


\section{REFERENCES}

[1] G. L. Matthaei, L. Young, and E. M. T. Jones, Microwave filters, impedance-matching networks, and coupling structures, Dedham Artech House, 1980

[2] D. Meledin et. al., "A 1.3-THz Balanced Waveguide HEB Mixer for the APEX Telescope", IEEE Trans. MTT, vol. 57, n. 1 , pp. $89-97,2009$.

[3] V. Desmaris, et. al, "All-metal micromachining for the fabrication of sub-millimetre and $\mathrm{THz}$ waveguide components and circuits," J. Micromech. Microeng., vol. 18, Art. ID $095004,2008$.

[4] D. Dochev, et. al. "A Technology Demonstrator for 1.6-2.0 THz Waveguide HEB Receiver with a Novel Mixer Layout", Int. J. Infrared Millim. Waves,vol. 32, 4, pp. 451-465, 2011.
[5] Pavolotsky, D. Meledin, C. Risacher, M. Pantaleev, and V. Belitsky, "Micromachining approach in fabricating of $\mathrm{THz}$ waveguide components," Microelectron. J., vol. 36, p. 636, 2005

[6] V. Belitsky et. al., "Facility Heterodyne Receiver for the Atacama Pathfinder Experiment Telescope," Proceedings of the IRMMW-THz 2007, Cardiff, UK, 2007.

[7] P. Mena et. al., "RF Performance of a 600 - 720 GHz SidebandSeparating Mixer with All-Copper Micromachined Waveguide Mixer Block," Proceedings of the ISSTT 2008, Groningen, The Netherlands, 2008. 